\title{
Analysis of Charge Distribution on Rectangular Microstrip Structures
}

\author{
E. Metlevskis* AND V. URBanavicius \\ Department of Electronic Systems, Vilnius Gediminas Technical University \\ Naugarduko str. 41-425, Vilnius LT-03227, Lithuania
}

\begin{abstract}
Rectangular microstrip structures are widely used in various microwave devices. Using the method of moments and principle of partial images, various techniques are developed to determine charge distribution in 2D models of microstrip structures (their cross-section). In this paper, a technique for calculating surface charge distribution and total capacitance of complex 3D rectangular microstrip structures is proposed using the mentioned method and principle. To demonstrate feasibility of the proposed technique, five rectangular microstrip structures were investigated. Obtained results are compared with the data published by other researchers. Total error is typically in $6-12 \%$ range.
\end{abstract}

PACS: 84.40.Az, 84.40.Lj, 41.20.Cv

\section{Introduction}

Rectangular microstrip structures (RMS) shown in Fig. 1 are widely used in various microwave devices, such as microstrip meander delay lines $[1,2]$, slow-wave deflecting systems [3], metamaterials forming [4, 5] etc. RMS are also successfully used for the analysis of physical models, for example, complex planar structures [6-8], microstrip discontinuities [9-11], etc.

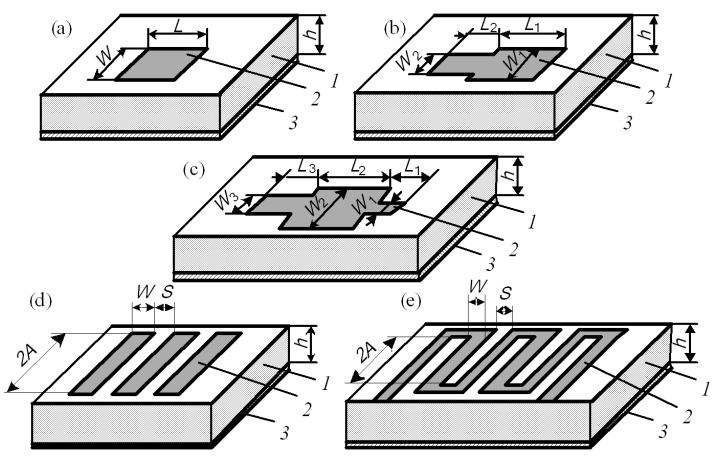

Fig. 1. Examples of rectangular microstrip structures: (a) the simplest microstrip segment, (b) microstrip with width joint, (c) microstrip with double width joint, (d) multiconductor microstrip line, (e) meander microstrip delay line: 1 - dielectric substrate, $2-$ microstrip section, 3 - ground plane.

Main characteristics of an RMS are charge distribution on the microstrip section or total capacitance of this sec-

* corresponding author; e-mail: edvardas .metlevskis@el.vgtu.lt tion. Other parameters, such as characteristic impedance and effective permittivity may be calculated from this distribution. Various analysis methods are used for calculation of these characteristics. Johnson et al. [4] use the simplest equation of the parallel plate capacitor

$$
C_{\mathrm{P}}=\varepsilon_{0} \varepsilon_{\mathrm{r}} A / h,
$$

where $\varepsilon_{0}$ is the dielectric constant of free space, $\varepsilon_{\mathrm{r}}$ is the relative dielectric permittivity of substrate of RMS, $A=W \times L$ is the area of the microstrip section of RMS, $h$ is the distance between the section and ground plane. While $A \gg h$ this approach is sufficiently accurate. However, increasing $h$ (e.g. using thick substrates) or decreasing $A$ (e.g. forming narrow microstrips) the inhomogeneous electric field on the RMS edges should be taken into account. Various approaches are used for the heterogeneity evaluation of the electric field. For example Das et al. [12] use conformal transformation, Ferrar and Adams [10] use matrix method, Nishiyama and Nakamura [13] use boundary elements method, Benedek and Silvester [14] solve Fredholm integral equation by projective method, empirically gotten expressions are also used [9] for this purpose.

In this paper a technique for analysis of charge distribution on RMS is proposed combining the method of moments (MoM) and principle of partial images $[15,16]$. This technique demonstrates rather fast computation speed and good accuracy.

\section{Proposed technique}

In order to find the charge distribution of the RMS we will use the advantages of the MoM and principle of the charges partial images that are in detail described in [15] 
for the 2D case, when the parameters per unit length of microstrip structure are calculated. A physical model of the simplest RMS for surface charge calculation is shown in Fig. 2. In the model, the microstrip section is divided into square sub-areas of size $a \times a$. We made premise that the charge on each sub-area is distributed uniformly. According to the example of model shown in Fig. 2, the number of sub-areas is 6 , so total length of the section is $L=2 a$ and width is $W=3 a$, respectively. Ground plane of the RMS is modeled placing the mirror charges $q_{m n}$ at a distance of $2 h$ from their original charges $q_{m n}$.

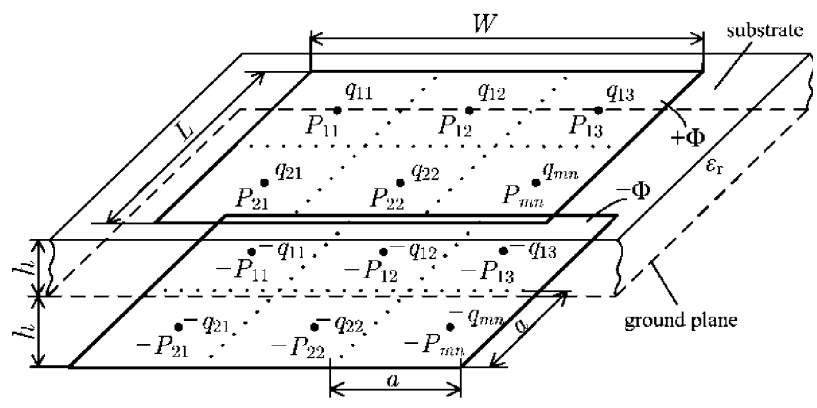

Fig. 2. Physical model of the rectangular microstrip section.

Mathematical model of RMS, based on the MoM, will consist of system of $N$ (number of sub-areas) linear equations which describe dependence of potentials $\phi_{i j}$ of sub-areas on their charge densities $\rho_{i j}$. Using the MoM and principle of partial images [15], the influence of every sub-area charge on the potential at the certain point of the RMS can be obtained by completing three steps:

Step 1. Find self-potential at point $P_{i i}$ that is raised by the charge $q_{i i}$ and its first partial image $K q_{i i}$ situated in the same point. It is shown in [17] that potential at the center of uniformly charged square-shaped plate of size $a \times a$ with density $\rho_{\mathrm{S} 0}$ can be calculated as

$$
\begin{aligned}
& \phi_{0,0}=a \rho_{\mathrm{S} 0}[\ln (1+\sqrt{2})] / \pi \varepsilon, \\
& \text { so } \phi\left(P_{i i}: P_{i i}\right)=a(1+K) \rho_{i i}[\ln (1+\sqrt{2})] / \pi \varepsilon_{0},
\end{aligned}
$$

where $K=-\left(\varepsilon_{\mathrm{r}}-1\right) /\left(\varepsilon_{\mathrm{r}}+1\right)$ is the reflection coefficient, $\rho_{i i}$ is the charge density of the $i i$ sub-area.

Step 2. Find potential that is raised by other partial images of charge $q_{i i}$

$$
\phi\left(P_{i i}: P_{i i}\right)=\frac{a^{2} K\left(1-K^{2}\right) \rho_{i i}}{4 \pi \varepsilon_{0}} \sum_{n=1}^{\infty} \frac{K^{2(n-1)}}{2 n h},
$$

where variable $n$ represents $n$-th partial image.

Step 3. Find potential which is raised by the mirror charge $-q_{i i}=-\rho_{i i} a^{2}$ and all its partial images which are situated in the point $-P_{i i}$ :

$$
\phi\left(P_{i i}:-P_{i i}\right)=\frac{a^{2} K\left(1-K^{2}\right) \rho_{i i}}{4 \pi \varepsilon_{0}} \sum_{n=1}^{\infty} \frac{K^{2(n-1)}}{(2 n-1) h} .
$$

To find a potential at point $P_{i j}$ that is raised by the charge $q_{m n}=\rho_{m n} a^{2}$ situated in the point $P_{m n}$, Eqs. (3)-(5) must be changed. Equation (3) changes to

$$
\phi\left(P_{i j}: P_{m n}\right)=\left[a^{2}(1+K) \rho_{m n}\right] R /\left(4 \pi \varepsilon_{0}\right),
$$

where $R=\sqrt{(i-m)^{2}+(j-n)^{2}}$ is the distance between points $P_{i j}$ and $P_{m n}$ expressed in $a$ units. Equation (4) in this case is changed to

$$
\begin{aligned}
\phi & \left(P_{i j}: P_{m n}\right)=-\frac{a^{2} K\left(1-K^{2}\right) \rho_{m n}}{4 \pi \varepsilon_{0}} \\
& \times \sum_{n=1}^{\infty} \frac{K^{2(n-1)}}{\sqrt{R^{2}+(2 n h)^{2}}},
\end{aligned}
$$

Eq. (5) is changed to

$$
\begin{gathered}
\phi\left(P_{i j}: P_{m n}\right)=-\frac{a^{2} K\left(1-K^{2}\right) \rho_{m n}}{4 \pi \varepsilon_{0}} \\
\times \sum_{n=1}^{\infty} \frac{K^{2(n-1)}}{\sqrt{R^{2}+[(2 n-1) h]^{2}}} .
\end{gathered}
$$

After the calculations in Eqs. (3)-(8) are performed, a matrix equation can be written

$$
\boldsymbol{\Phi}=\boldsymbol{A} \times \boldsymbol{q},
$$

where the element $A_{i j}$ of matrix $\boldsymbol{A}$ represents the potential of $i$-th sub-area that is raised by the charge in the $j$-th sub-area, $\boldsymbol{\Phi}$ is the column vector of known potentials sent into sub-areas, $\boldsymbol{q}$ is the column vector of unknown charges. Unknown charges are obtained solving Eq. (9), e.g. using the inverse matrix $\boldsymbol{A}^{-1}$

$$
\boldsymbol{q}=\boldsymbol{A}^{-1} \times \boldsymbol{\Phi} .
$$

It should be noted that if the potential difference between plates is $2 \mathrm{~V}$, the total capacitance of the RMS is

$$
C=\sum_{i=1}^{N} q_{i} / 2
$$

where $N$ is a total number of RMS sub-areas.

\section{Results of the analysis of rectangular microstrip structures}

To validate the technique proposed in this paper, normalized capacitances of the simplest RMS (see Fig. 1a) were calculated and compared with the results of other researchers [10] and [14]. For this purpose, using Eqs. (2)-(11), a program was written to calculate the capacitance of RMS shown in Fig. 1a-c. The number of sub-areas $N$ of the analyzed RMS was correspondingly 500, 800, and 1200. The infinity in Eqs. (4), (5), (7), and (8) was restricted by 50 partial images. It was determined that the larger number of images does not 
increase calculation accuracy. Results of computations are presented in Fig. 3-6.

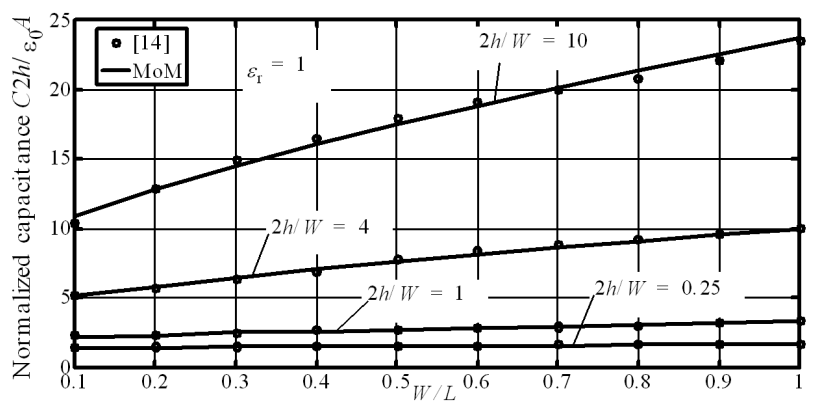

Fig. 3. Calculated normalized capacitance of the simplest microstrip segment (see Fig. 1a) for relative dielectric permittivity of the substrate $\varepsilon_{\mathrm{r}}=1$.

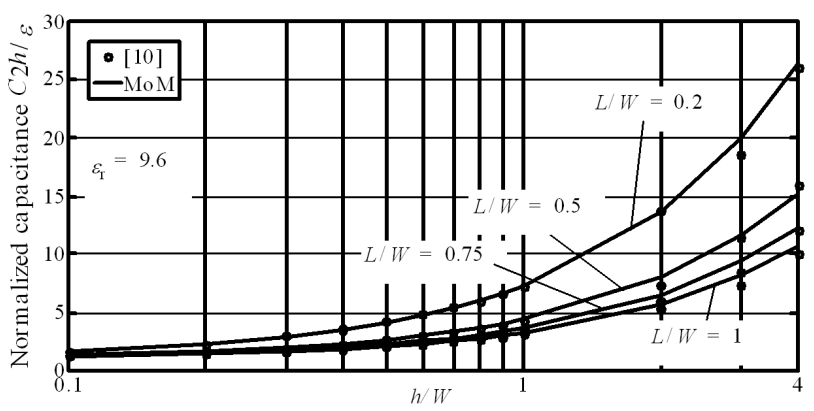

Fig. 4. Calculated normalized capacitance of the simplest microstrip segment (see Fig. 1a) for relative dielectric permittivity $\varepsilon_{\mathrm{r}}=9.6$.

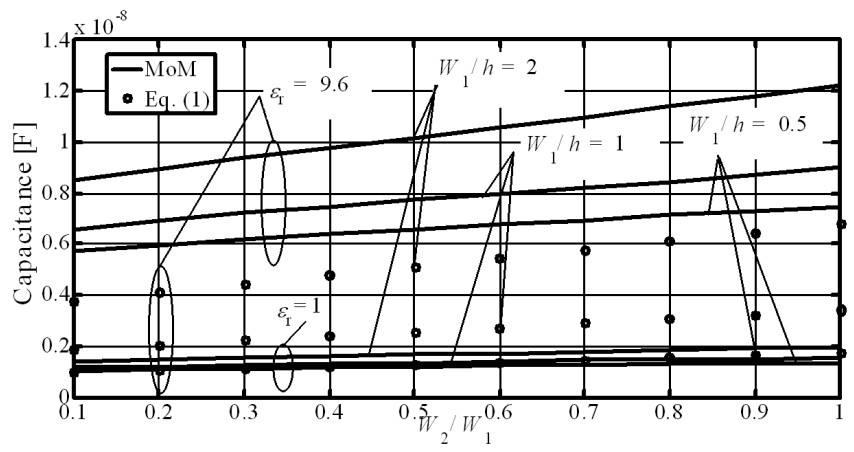

Fig. 5. Total capacitance of microstrip with width joint (see Fig. 1b) for $\varepsilon_{\mathrm{r}}=1, \varepsilon_{\mathrm{r}}=9.6$, and $W_{1} / h=2$, $W_{1} / h=1, W_{1} / h=0.5$.

Normalized capacitance of the simplest microstrip segment (see Fig. 1a) for various $W / L$ ratios is presented in Fig. 3. Here relative dielectric permittivity of the substrate $\varepsilon_{\mathrm{r}}=1$ and various distances between sections-to-width $2 h / W$ ratios were selected to compare calculated results with data published in [12]. It is seen in Fig. 3 that these results agree within $5 \%$.

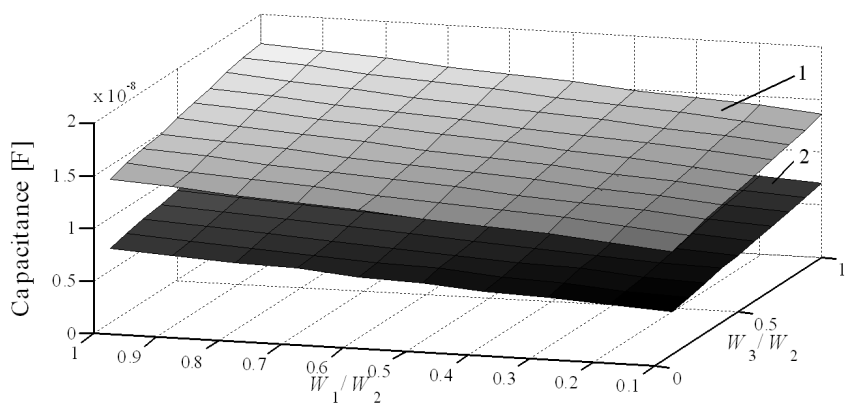

Fig. 6. Total capacitance of microstrip with double width joint (see Fig. 1c) for $\varepsilon_{\mathrm{r}}=9.6$ and $W_{2} / h=2$. 1 - calculations performed using proposed technique, 2 - calculations performed according to parallel plate capacitor Eq. (1).

Slightly different calculation results are shown in Fig. 4. Here, normalized capacitance of simplest microstrip section (see Fig. 1a) depends on $h / W$ ratios. For permittivity of the substrate $\varepsilon_{\mathrm{r}}=1$ and $h / W$ ratios $\leq 1$, calculations agree with [10] within $6 \%$. For greater $h / W$ ratios $(h / W>1)$, these results agree within $12 \%$. This is rather large difference and may be explained by the fact that mathematical model used in work [10], to calculate the capacitance of RMS, was realized using quite different equations describing relationships between potentials and charges, so obtained percentage difference is acceptable.

Using the proposed technique, dependences of capacitances of complex RMS, which are shown in Fig. 1b and Fig. 1c, on their design parameters, were also investigated. Calculation results are shown in Fig. 5 and Fig. 6.

Dependences of the total capacitance of the RMS with one width joint (see Fig. 1b) on width $W_{2}$ and $\varepsilon_{\mathrm{r}}$ are shown in Fig. 5. Performing the computations, width $W_{1}$ was set to constant so that $W_{1}=L_{1}=L_{2}$. It is seen in Fig. 5 that the capacitance of RMS increases when the width $W_{1}$ increases also. During our investigation several different relative dielectric permittivities $\varepsilon_{\mathrm{r}}$ and thicknesses of the substrate (expressed by $W_{1} / h$ ratios) were selected. It should be noted that the higher total capacitance is received for the larger $\varepsilon_{\mathrm{r}}$ values and $W_{1} / h$ ratios. It is also seen in Fig. 5 that the total capacitance of the RMS, which has the substrate with larger $\varepsilon_{\mathrm{r}}$, increases, with increasing $W_{2} / W_{1}$, more quickly.

To demonstrate the possibility of the proposed technique to take into account heterogeneity of the electric field on the RMS edges, dependences of the total capacitance, calculated according to Eq. (1) in Fig. 5 are also shown (by dots). It is seen that capacitances calculated by the proposed technique are larger than those calculated by Eq. (1). The thicker the substrate the bigger difference between capacitances is received (1.8-2.2 times for $W_{1} / h=2$, and $2.8-5.8$ times for $\left.W_{1} / h=0.5\right)$. As a result of investigation, it is seen that the proposed technique takes into account the heterogeneity of the electric 
field on the RMS edges.

Results of the analysis of the RMS with double width joint (see Fig. 1c) are shown in Fig. 6. For more comfortable representation of calculated data, 3D graph was drawn. Similarly to Fig. 5, during the computations width $W_{2}$ and distance to ground plane $h$ were set to constant so that $W_{2}=L_{1}=L_{2}=L_{3}$ and $W_{2} / h=2$. It is seen that the increased width $W_{1}$ and $W_{3}$ increases total capacitance of the structure almost linearly. This can be explained by the increased area of microstrip section: as width $W_{1}$ and $W_{3}$ gets wider, the structure approaches rectangular shape. The possibility of proposed technique to evaluate the scattering of the electric field on the RMS edges in Fig. 6 is demonstrated, too. The 1st surface in Fig. 6 corresponds to the total capacitance of the RMS calculated by the proposed technique, and the 2nd surface corresponds to the capacitance calculated accordingly to Eq. (1).

\section{Research of multiconductor microstrip line}

During the analysis of the multiconductor microstrip lines - MCML (see Fig. 1d) it is often assumed that the length of the conductor is infinite. In such case the surface charge distribution is investigated only in cross-section (2D MCML model) [18]. Infinite length MCML can be applied in the creation of mathematical models for the devices with periodical structures [19]. The main goal of our research is to calculate the surface charge distribution in different parts of 3D MCML and determine the length of MCML (ratio $A / h$ ) such that $3 \mathrm{D}$ model of MCML approaches the results obtained by $2 \mathrm{D}$ model of MCML. The calculated surface charge distribution of 3D MCML model is shown in Fig. 7. In 2D model of MCML it is considered that the surface charge distribution at any given cross-section in the direction of $y$ axis is the same. For that purpose, a special program for determination of surface charge distribution in 2D MCML (its cross-section) was also written.

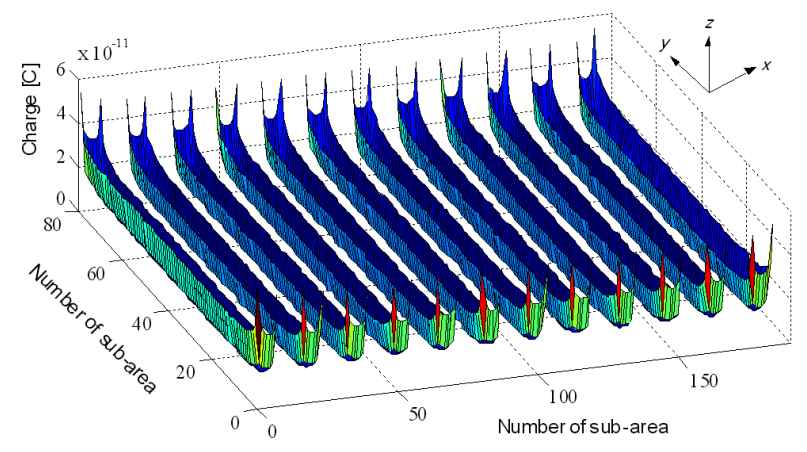

Fig. 7. 3D surface charge distribution on microstrip multiconductor line.

Surface charge distribution of different cross-sections of MCML is shown in Fig. 8. During the computations, a line consisting of 12 conductors, was designed. Each conductor was divided into 8 sub-areas in the $x$-direction and into 80 sub-areas in the $y$-direction. The width of the conductor was set to $W=S$. Drawing the charge distribution of 3D model, cross-section was selected in the middle (see Fig. 8a) and in the ends (see Fig. 8b) of the conductors as those cross-sections appears where the ports in real devices are usually placed. It is seen that with increase of a length of MCML conductor, surface charge distribution of $3 \mathrm{D}$ model approaches the results obtained by $2 \mathrm{D}$ model.

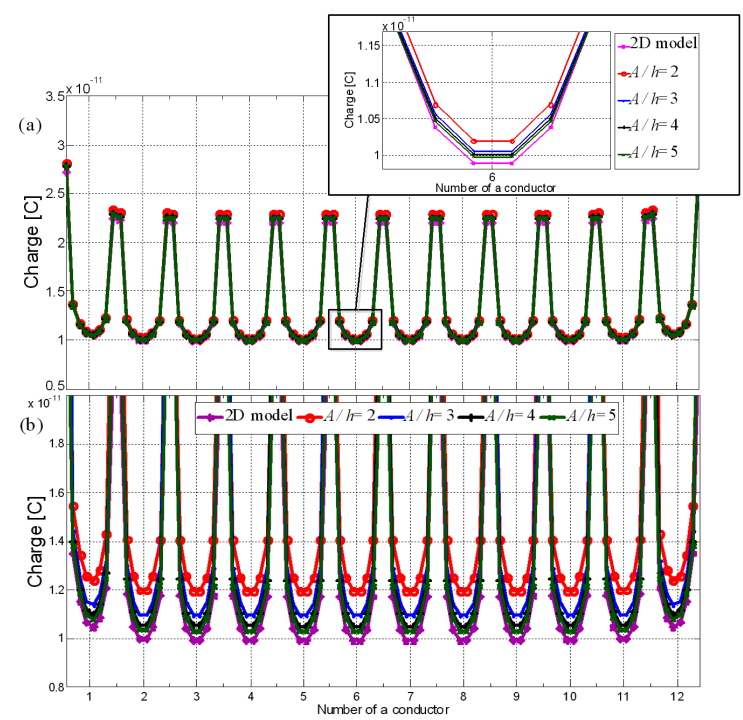

Fig. 8. Charge distribution in the cross-section of MCML: cross-section selected in the middle of the conductors (a) and in the ends of the conductors (b).

Difference between 2D and 3D models of MCML can be shown by comparing relative difference of surface charge distribution which was calculated in the middle of the conductors relatively to $x$-axis. It is determined that according to Fig. 8a, lowest difference value is received for the longest investigated MCML $(A / h=5)$ in the 1 st conductor and equals $0.57 \%$, while highest difference value is received for the shortest investigated MCML $(A / h=2)$ in the 6 th conductor and equals $3 \%$. Similar results are obtained for Fig. 8b with the lowest and the highest difference values of $3 \%$ and $21 \%$, respectively.

\section{Modeling of meander delay line}

To analyze the surface charge distribution in complex microstrip structure, a model of meander microstrip delay line was proposed. The structure of a delay line, shown in Fig. 1e, is based on the multiconductor line consisting of 12 conductors that was designed in Sect. 4 . The design parameters of the delay line are: $A / h=4$, $W / h=1, S / h=0.25$. Similarly as in multiconductor line, strip was divided into 8 sub-areas along $x$-axis and into 80 sub-areas along $y$-axis. Surface charge distribution, when the potential of $+1 \mathrm{~V}$ is applied to the meander conductor, is shown in Fig. 9. It is seen that bigger 
charge is concentrated on the side strips of the meander. Moreover, sudden increase of a charge on the edges of the conductor has to be taken into account.

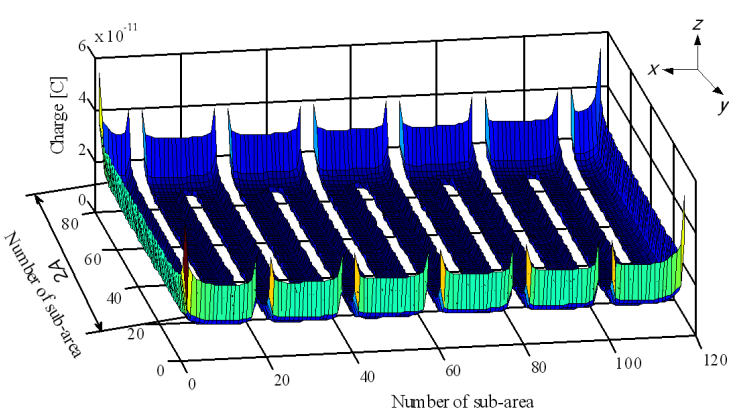

Fig. 9. Surface charge distribution on meander delay line.

Surface charge distribution of the meander conductor fragment where two strips are connected is shown in Fig. 10. Here the charge distribution on the inner corners of meander and side strip is brought on. It is seen that charge on the inner corner of meander decreases. This can be explained by the charges of the same sign which are situated on the adjacent strip edges in such way that they reduce each other. Also, Fig. 10 shows a clearer view of charge distribution on a single strip. It can be seen that the charges concentrates on the strip edges. Such concentration of the charges can be explained by the fact that a potential of $+1 \mathrm{~V}$ is applied to a delay line and the charges of the same sign displace each other to the edges of a conductor.

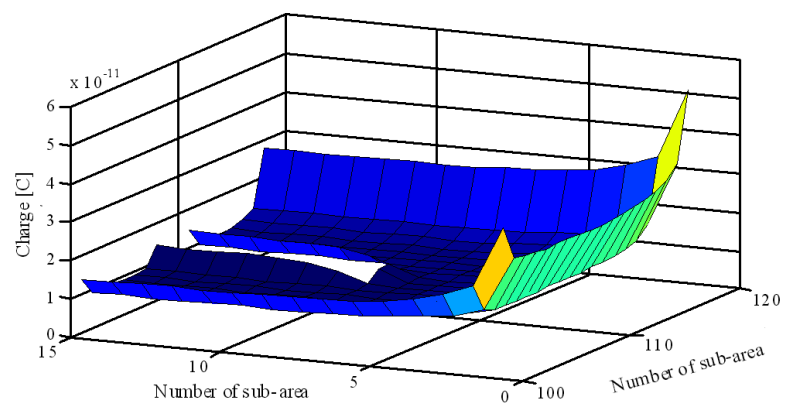

Fig. 10. Surface charge distribution of meander conductor fragment where two strips are connected.

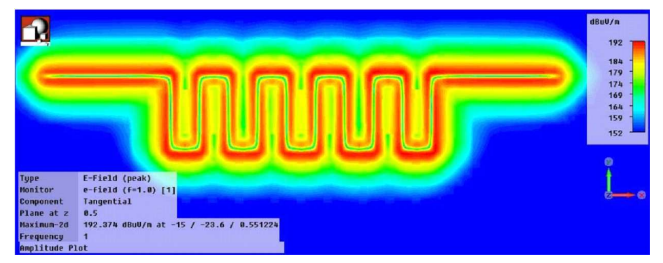

Fig. 11. Distribution of the electric field magnitude in the meander delay line [20].
Obtained charge distributions can be compared with the results in paper [20] where the dependences of the meander line time delay parameters are analyzed. In Ref. [20], the system is analyzed using $1 \mathrm{GHz}$ signal. Distribution of electric field in this meander delay line is shown in Fig. 11. It is seen that the stronger electric field is also concentrated on the edges of a strip, whereas on the inner corners electric field is much weaker. Although the meander line shown in Fig. 11 has large gaps between conductors $(S / h=10)$, distribution is somewhat similar to the results shown in Fig. 9. So the analysis method proposed in this paper can also be used to calculate the charge distribution in the quasi-static approach on the surface of complex planar structures.

\section{Conclusion}

RMS are widely used in various microwave devices. Using the method of moments and principle of partial images, various techniques are developed to determine charge distribution in 2D models of microstrip structures (their cross-section). In this paper, a technique for calculating surface charge distribution and total capacitance in complex 3D rectangular microstrip structures is proposed using the mentioned method and principle. To demonstrate feasibility of the proposed technique authors have written the specific program and investigated five RMS. The investigated RMS were divided into 500-1200 sub-areas, the sufficient number of the partial charge images was set to 50. Calculation time, depending on the analyzed structure, has varied from $19 \mathrm{~s}$ to $152 \mathrm{~s}$ (CPU clock was $1.73 \mathrm{GHz}$, and RAM size was $1 \mathrm{~GB}$ ). Obtained results are compared with the data published by other researches. Total error is typically less than 5\%. Also a multiconductor microstrip line was investigated. 3D and 2D models of MCML were compared by calculating the relative differences of the surface charge distributions in different cross-sections of the line. Highest difference values for the cross-sections in the middle and at the ends of the line are $3 \%$ and $21 \%$, respectively. Moreover, microstrip meander delay line was modeled. The surface charge distribution when the potential of $+1 \mathrm{~V}$ is applied to the meander conductor is discussed.

\section{References}

[1] V. Daškevičius, J. Skudutis, S. Štaras, Electron. Electr. Eng. 74, 37 (2007).

[2] V. Urbanavičius, A. Gurskas, R. Martavičius, Electron. Electr. Eng. 90, 6 (2009).

[3] V. Daškevičeius, J. Skudutis, S. Štaras, Electron. Electr. Eng. 89, 101 (2009).

[4] N.P. Johnson, A.Z. Khokhar, H.M.H. Chong, R.M. De La Rue, S. McMeekin, Electron. Lett. 42 , 1117 (2006).

[5] L. Nickelson, T. Gric, S. Ašmontas, R. Martavičius, Electron. Electr. Eng. 93, 87 (2009). 
[6] A. Casanueva, E. Zubizarreta, O. González, A. Grande, E. Villa, in: Proc. 1st Seminar - Portuguese Committee of URSI, Radiocommunications - New Paradigms and Health Impact, Lisbon $200 \%$. Available online: http://www.anacom.pt/streaming/ Analysis LPF Structure.pdf?categoryId $=260643$ \&contentI $\bar{d}=542 \overline{7} 57 \&$ field $=$ ATTACHED FILE

[7] C.Y. Kim, Prog. Electromagn. Res. B 8, 179 (2008).

[8] L. Nickelson (Knisevskaja), M. Tamosiuniene, V. Shugurov, Int. J. Infrared Millimeter Waves $\mathbf{2 3}$ 325 (2002).

[9] G. Stewart, M. Kay, C.H. Riedell, R. Pomerleau, M. Steer, Southeastcon '89. Proceedings IEEE 1, 107 (1989).

[10] A. Farrar, A.T. Adams, IEEE Trans. Microw. Theory Tech. 20, 497 (1972).

[11] F. Huang, C.H. Loh, C.H. Poh, Int. J. RF Microwave Computer-Aided Eng. 8, 367 (1998).

[12] B. Das, S. Chakrabarty, K. Siva Rama Rao, IEEE Trans. Electromag. Compabil. 37, 94 (1995).

[13] H. Nishiyama, M. Nakamura, IEEE Trans. Compon. Package Manufact. Technol. Part A 17, 477 (1994).
[14] P. Benedek, P. Silvester, IEEE Trans. Microw. Theory Tech. 20, 504 (1972).

[15] V. Urbanavičius, Š. Mikučionis, R. Martavičius, Electron. Electr. Eng. 77, 23 (2007).

[16] E. Metlevskis, V. Urbanavičius, in: Proc. 18th Int. Conf. Microwave Radar and Wireless Communications (MIKON), 2010, p. 228. Available online: http://ieeexplore.ieee.org/stamp/stamp.jsp?tp= \&arnumber $=5540460$.

[17] N.N. Rao, Elements of Engineering Electromagnetics, Prentice Hall, New Jersey 1994.

[18] V. Urbanavičius, R. Pomarnacki, in: Proc. 18th Int. Conf. Electromagnetic Disturbances EMD, Technika ISSN 1822-3249, Vilnius 2008, p. 203.

[19] T. Burokas, S. Staras, Electron. Electr. Eng. 84, 3 (2008).

[20] S. Wei-Shan, S. Kye-Yak, R.W.-Y. Chang, M. Oswal, W. Ling-Biao, in: Proc. IEEE Asia Pacific Microwave Conf, Nanyang Technol. Univ., Singapore 2009, p. 1285 . 